\title{
Nearly constant loss or constant loss in ionically conducting glasses: A physically realizable approach
}

\author{
J. Ross Macdonald ${ }^{\text {a) }}$ \\ Department of Physics and Astronomy, University of North Carolina, Chapel Hill, \\ North Carolina 27599-3255
}

(Received 3 April 2001; accepted 10 July 2001)

Conductivity exhibiting power-law frequency response with an exponent of unity leads to frequency-independent dielectric loss. Such constant-loss (CL) behavior is not physically realizable over a nonzero frequency range, and approximate expressions that have been used to represent it are inconsistent with the Kronig-Kramers relations. Response models are proposed and investigated that do satisfy these relations and can lead to very close approximation to CL over many frequency decades, as often observed at low temperatures in ionic conductors such as glasses. Apparent CL response is shown to arise from the series connection of a constant-phase complex-power-law element $(\mathrm{CPE})$, with exponent $\delta(0<\delta \ll 1)$, and a frequency-independent dielectric constant, $\varepsilon_{U}$. Two physically disparate situations can lead to such a series connection. The first involves bulk CPE response in series with an electrode-related, double-layer blocking capacitance involving a dielectric constant $\varepsilon_{S}$. Then, apparent CL behavior may be associated with localized ionic motion in the bulk of the material. The second (mirror-image) situation involves CPE response associated with ionic motion in or at an electrode in series with a capacitance such as the bulk high-frequency-limiting total dielectric constant $\varepsilon_{\infty}$ or the pure-dielectric quantity $\varepsilon_{D_{\infty}}$. The present model is used to simultaneously fit both the real and imaginary parts of data derived from measurements on a sodium-trisilicate glass at $122 \mathrm{~K}$. This data set exhibits power-law nearly constant loss for $\varepsilon^{\prime}(\omega)$ and apparent CL for $\varepsilon^{\prime \prime}(\omega)$. The magnitude of the CL closely satisfies a simple equation involving only $\delta$ and $\varepsilon_{U}$. Further, for the electrode-power-law situation, estimated values of limiting-high-frequency dielectric constants turn out to be more consistent with bulk values established at much higher temperatures where nearly constant loss is no longer a dominant part of the response. Data at $-0.5^{\circ} \mathrm{C}$ are also analyzed with a more complicated composite model, one that is a generalization of both of the above approaches, and nearly constant loss bulk, not electrode, power-law effects in both $\varepsilon^{\prime}(\omega)$ and $\varepsilon^{\prime \prime}(\omega)$ are isolated and quantified. For this data set it is shown that electrode effects are important at both ends of the frequency range. (C) 2001 American Institute of Physics. [DOI: 10.1063/1.1398299]

\section{INTRODUCTION AND BACKGROUND}

The field of impedance (or more generally, immittance) spectroscopy (IS), the measurement and analysis of the frequency and temporal electrical response of solids and liquids, has burgeoned in the last decade. This growth has occurred both because IS data are useful for helping one understand the basic electrical processes present in the material considered and because IS has proved valuable in many applied areas, such as process control and characterization. Although complex-nonlinear-least squares (CNLS) fitting of IS data allows one to take proper account of both bulk and electrode contributions to the data, required for accurate estimation of the parameters of a fitting model, much data continue to be analyzed using a model that represents only bulk response associated with mobile ions.

An important and puzzling feature of much IS data for ion-conducting glasses is the presence of apparent constant loss (CL) or nearly constant loss (NCL) effects, those where

a)Electronic mail: macd@email.unc.edu the electrical loss is entirely or nearly frequency-independent, respectively. In recent work, $\mathrm{Ngai}^{1}$ presented a valuable comprehensive survey of apparent CL behavior for a variety of materials and material factors, and he characterized CL as a spectacular phenomenon because of its ubiquity. Although he stated that the physical mechanisms leading to CL were uncertain, he suggested that for ionic conductors CL is associated with ionic librational or vibrational motion. Earlier, Nowick and co-authors ${ }^{2,3}$ somewhat similarly interpreted CL effects as arising from dielectric dispersion associated with the local motion of ions.

There seems to be considerable current agreement that NCL in ionically conducting materials is associated with vibration and relaxation of ions confined locally in potential wells, ${ }^{1,3-6}$ but a related suggestion is that CL arises from low-energy distortions of the ionic network. ${ }^{7}$ Most importantly, however, none of these suggestions has yet resulted in a quantitative theory predicting the details of the NCL behavior. Although a descriptive model should predict and fit both parts of the full complex dielectric constant, $\varepsilon(\omega)$ 
$=\varepsilon^{\prime}(\omega)-i \varepsilon^{\prime \prime}(\omega)$, most NCL experimental results have been presented only in terms of $\varepsilon^{\prime \prime}(\omega)$, often without much or any correct consideration of its associated Kronig-Kramers (KK) pair, $\varepsilon^{\prime}(\omega)$.

In 1991, Lee, Liu, and Nowick ${ }^{8}$ suggested that a frequency-independent contribution to the dielectric loss of ionically conducting crystals and glasses was universally present. Since then, the following composite-model expression has frequently been used to describe the full response of the real part of the complex conductivity (e.g., Ref. 3),

$$
\sigma^{\prime}(\omega)=\left[\sigma_{0}+a \omega^{S_{B}}\right]+A \omega^{S},
$$

where all five parameters are taken frequency independent and $0<S_{B} \leqslant 1$. Here $\sigma(\omega)=\left[i \omega \varepsilon_{V} \varepsilon(\omega)\right]=\sigma^{\prime}(\omega)$ $+i \sigma^{\prime \prime}(\omega) ; \sigma_{0} \equiv \sigma^{\prime}(0)$; and $\varepsilon_{V}$ is the permittivity of vacuum.

The $A \omega^{S}$ part of Eq. (1) leads to a frequencyindependent term in $\varepsilon^{\prime \prime}(\omega)$ when $S=1$, thus representing constant loss. The term in square brackets, which represents bulk mobile-ion response, has been called universal dynamic response, ${ }^{3}$ but it is actually not all that universal and has been shown to be a poor choice for describing bulk dispersion. ${ }^{9}$ To investigate NCL or CL response present in experimental data, it is clear that in general one should fit such data with a composite fitting model that includes a bulk response model and at least one other term that can represent NCL behavior, but these contributions need not necessarily be in parallel as in Eq. (1). Note, however, that at sufficiently low temperatures it is often observed that a NCL term sufficiently dominates the response that no other terms need be included in the fitting model. This matter is further examined herein.

In 1994, no CL behavior was found ${ }^{10}$ for single-crystal $\mathrm{NaCl}$, and later analysis ${ }^{11}$ showed that the NCL expression, $S=1-\left(T / T_{0}\right)$, applied quite accurately for the lowtemperature region of $\mathrm{CaTiO}_{3} \cdot 30 \% \mathrm{Al}^{3+}$ data down to at least $S \simeq 0.94$, in accordance with theoretical predictions. ${ }^{12}$ Perhaps these results led some workers in the field to refer in subsequent work to NCL rather than to CL. ${ }^{3,13}$ We further investigate this distinction herein. In a recent important contribution, which deals primarily with the transition from NCL to ordinary bulk response, ${ }^{6}$ Leon and co-authors point out that their results preclude the applicability of the sum-ofresponses model represented by Eq. (1). The present results quantify this conclusion while raising important questions about the contribution of ions to NCL.

By using the approximate expression ${ }^{14}$

$$
\varepsilon^{\prime \prime}(\omega) \simeq-(\omega \pi / 2)\left[\mathrm{d} \varepsilon^{\prime}(\omega) / \mathrm{d} \omega\right],
$$

Ngai ${ }^{1}$ obtained a formula for the corresponding $\varepsilon^{\prime}(\omega)$ when $\varepsilon^{\prime \prime}(\omega)$ was taken frequency independent. He then employed his results to eliminate apparent CL effects from $\mathrm{Na}_{2} \mathrm{O} \cdot 3 \mathrm{SiO}_{2}$ glass data at $-0.5^{\circ} \mathrm{C}$, but this approach is incomplete and does not lead to valid estimates of all bulkmodel parameters. ${ }^{9}$ In this work, Ngai used the wellknown original modulus formalism ${ }^{15}$ (OMF) to represent conductive-system bulk response, but it makes no distinction between $\varepsilon_{\infty}$, the high-frequency-limiting effective dielectric constant arising from all sources, and $\varepsilon_{C 1 \infty}$, that part of $\varepsilon_{\infty}$ arising solely from mobile charge effects. An alternate analysis of this data set is presented in Sec. III B.

As shown elsewhere, ${ }^{9,16-18}$ one needs to correct the OMF approach by defining its high-frequency-limiting dielectric response as $\varepsilon_{C 1 \infty}$ and separately including in a full fitting model the important quantity $\varepsilon_{D^{\infty}}$, the highfrequency-limiting dielectric constant arising from dipolar and vibratory motions of the nonionic bulk constituents of the material with or without mobile ions present. It follows that $\varepsilon_{\infty} \equiv \varepsilon_{C 1 \infty}+\varepsilon_{D \infty}$. The resulting corrected modulus formalism (CMF), like the OMF, involves a KohlrauschWilliams-Watts, KWW1, frequency-response model associated with mobile charge and derived from stretchedexponential temporal response. ${ }^{9,16,17,19}$ This model, designated by $\mathrm{K} 1$, will be used herein. It involves a shape parameter $\beta_{1}$.

Although it is desirable that present analysis methods be applied to some of the same low-temperature $\mathrm{Na}_{2} \mathrm{O} \cdot 3 \mathrm{SiO}_{2}$-glass numerical data sets analyzed in Ref. 3, this has proved to be impossible. Professor Nowick has informed me that these wide-temperature-range data sets cannot be found. On the other hand, the $\mathrm{T}=-0.5^{\circ} \mathrm{C}$, $\mathrm{Na}_{2} \mathrm{O} \cdot 3 \mathrm{SiO}_{2}$ set used in the CL discussion and analysis of Ref. 1 has been kindly sent to me by Professor C. T. Moynihan and will be analyzed in Sec. III B.

In the absence of direct numerical data for the lowtemperature region where NCL effects are most dominant, the $\mathrm{Na}_{2} \mathrm{O} \cdot 3 \mathrm{SiO}_{2}$ graphical results for $\varepsilon(\omega)$ at $122 \mathrm{~K}$ presented in Fig. 8 of Ref. 3 will be employed. The $\varepsilon^{\prime}(\omega)$ curve is very closely of power-law character, and its values were accurately scaled from the graph. The corresponding $\varepsilon^{\prime \prime}(\omega)$ response is very close to $\mathrm{CL}$ over an appreciable frequency range. For this $\varepsilon(\omega)$ data set, like other similar lowtemperature ones, ${ }^{3}$ bulk-dispersion loss arising from any long-range motion of the ions generally falls well below that associated with other processes, as discussed below. Although one cannot directly fit and estimate the parameters of such a bulk dispersion model at low temperatures such as $122 \mathrm{~K}$, extrapolation from higher temperatures, where good estimates of such parameters are available from K1 fitting, ${ }^{9,17}$ allows semi-quantitative evaluation of the contribution of ionic motion to the response at these temperatures.

The qualifier "apparent" has been used above in discussing possible CL behavior. As shown below, it seems unlikely that ideal nonzero CL response is possible over a finite frequency range. Nevertheless, such a close approximation to CL may occur experimentally that it cannot be distinguished from true CL because of the presence of unavoidable data errors. I shall therefore use CL herein to indicate such apparent constant loss.

In the following sections, two different types of NCL and CL response functions will be defined and compared with other approaches that have been used in the past to represent such behavior. Accurate data fitting using the LEVM complex-nonlinear-least-squares computer program ${ }^{20}$ will be shown to allow discrimination between the various choices. Finally, some higher-temperature results, where bulk dispersion effects arising from long-range ionic motion must 
be explicitly included in the fitting model, will be analyzed using the physically realistic NCL response functions considered here.

\section{NEARLY-CONSTANT-LOSS FITTING MODELS}

\section{A. Parallel nearly-constant-loss model}

The first fitting element to be considered is a constantphase-element (CPE) expression ${ }^{21,22}$ defined at the complex dielectric constant level by

$$
\varepsilon_{\mathrm{PC}}(\omega)=A_{\mathrm{PC}}(i \omega)^{-\gamma_{\mathrm{PC}}}=A_{\mathrm{PC}} \omega^{-\gamma_{\mathrm{PC}}[\cos (\psi)-i \sin (\psi)]},
$$

where $\psi \equiv\left(\gamma_{\mathrm{PC}} \pi / 2\right)$. This CPE power-law response function, whose parts satisfy the KK relations, will be designated as PCPE or P and will taken as a bulk rather than electrode response. Note that when $|\psi| \ll 1$, the $\omega$ dependence in Eq. (3) is very small, $A_{\mathrm{PC}}$ is very close to being an ideal dielectric constant, and $\varepsilon_{P C}^{\prime \prime}(\omega)$ is a NCL term. It is readily shown that the approximate Eq. (2) expression holds well for the real and imaginary parts of $\varepsilon_{\mathrm{PC}}(\omega)$ when $\gamma_{P C}^{2} \ll 1$ and $\gamma_{\mathrm{PC}}$ $\neq 0$. The subscript "PC" is used here to indicate that the NCL response is in parallel electrically with any other conductive-system bulk response, conventionally defined at the complex resistivity level. In practice, real data always includes contributions from $\varepsilon_{D^{\infty}}$, so the effects of this nonionic dielectric constant must be properly accounted for in fitting experimental data, as discussed and illustrated in detail in Sec. III.

Equation (3) leads to constant loss when $\gamma_{\mathrm{PC}}=0$, but the loss is then of zero magnitude. The condition $\gamma_{\mathrm{PC}}<0$ yields a negative loss, or gain, and contributes a corresponding negative increment to $\sigma^{\prime}(\omega)$. Such response is at the least unlikely and is physically impossible for a passive linear system. These considerations indicate that when fits include the PCPE, valid estimated values of $\gamma_{\mathrm{PC}}$ should always be positive or zero. The $\gamma_{\mathrm{PC}}=0$ condition is equivalent to the addition to the model of a pure capacitance, such as that represented in the present work by $\varepsilon_{x}$.

Note that if a nonzero constant loss, $B_{0}$, were possible for a finite frequency range, Eq. (2) would then lead to the expression

$$
\varepsilon_{\mathrm{CL}}(\omega)=-(2 / \pi) B_{0} \ln \left(\omega / \omega_{0}\right)-i B_{0},
$$

where $\varepsilon_{\mathrm{CL}}^{\prime}(\omega)$ is positive and decreasing with increasing $\omega$ so long as $\omega_{0}>\omega$. Clearly $\varepsilon_{\mathrm{CL}}^{\prime}(\omega)$ increases indefinitely as $\omega \rightarrow 0$. Although Eq. (4) was not explicitly presented by Ngai, ${ }^{1}$ it was evidently used in his work, and Eq. (2) was also employed in the earlier NCL analysis of Ref. 3. It is therefore worthwhile to compare the differences between the fitting appropriateness of the $\varepsilon_{P C}^{\prime}(\omega)$ of Eq. (3) and the $\varepsilon_{C L}^{\prime}(\omega)$ of Eq. (4).

Accurate scaling of the $122 \mathrm{~K} \mathrm{Na}_{2} \mathrm{O} \cdot 3 \mathrm{SiO}_{2} \varepsilon^{\prime}(\omega)$ data presented in Fig. 8 of Ref. 3 and least-squares fitting of the resulting estimated values by the $\varepsilon_{P C}^{\prime}(\omega)$ part of the powerlaw model of Eq. (3) led to PCPE parameter estimates of $A_{\mathrm{PC}}=8.885$ and $\gamma_{\mathrm{PC}}=0.00320$. From these values, one can use Eq. (3) to calculate the full $\varepsilon^{\prime}(\omega)$ and $\varepsilon^{\prime \prime}(\omega)$ data curves, ones that satisfy the KK relations. Exact results are

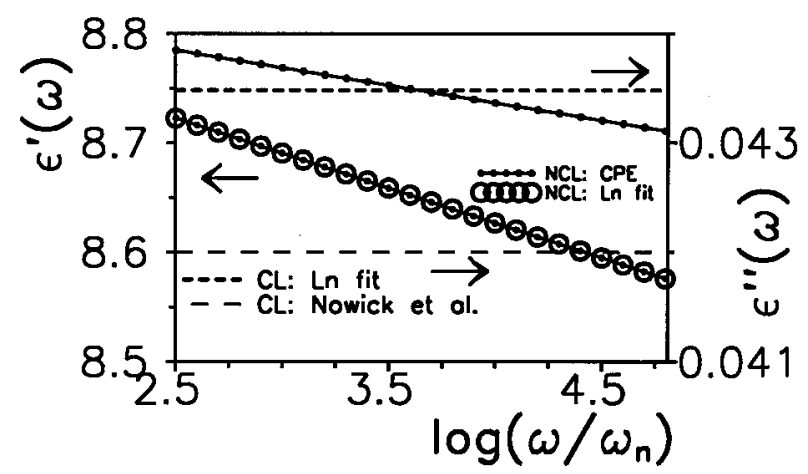

FIG. 1. The two lines designated NCL: CPE are exact $\varepsilon^{\prime}(\omega)$ and $\varepsilon^{\prime \prime}(\omega)$ results derived from fitting the $\varepsilon^{\prime}(\omega)$ data of Ref. 3, whose CL $\varepsilon^{\prime \prime}(\omega)$ $=B_{0}=0.042$ estimate is shown as the bottom dashed horizontal line. The $\varepsilon^{\prime}(\omega)$ NCL: Ln points are the results of fitting the $\varepsilon^{\prime}(\omega)$ NCL: CPE data with Eq. (4) of the text. Such fitting led to the $B_{0} \simeq 0.0435$ value shown as the upper dashed horizontal line. Here and elsewhere $\omega_{n}=1 \mathrm{r} / \mathrm{s}$.

shown in Fig. 1 by the two straight lines with solid-circle data points designated by NCL: CPE. Forty-seven points were used to cover the range of about 2.3 decades shown in Ref. 3.

Also shown in Fig. 1 is the fit of the $\varepsilon_{P C}^{\prime}(\omega)$ curve by Eq. (4), that designated as NCL: Ln fit. The fit appears perfect on the present scale but actually involved a value of the relative standard deviation of the fit, $\mathrm{S}_{\mathrm{F}}$, of about $10^{-5}$. Parameter estimates for this fit were about $B_{0}=0.0435$ and $\ln \left(\omega_{0} / \omega_{n}\right)=320.9$. Here $\omega_{n}$ is just $1 \mathrm{r} / \mathrm{s}$, a normalization parameter. Although the $\varepsilon_{P C}^{\prime}(\omega)$-model parameter estimates were slightly different and $\mathrm{S}_{\mathrm{F}}$ was about $5 \times 10^{-5}$ for data extending from 1 to $10^{5} \mathrm{r} / \mathrm{s}$, the present results indicate that for such a small value of $\gamma_{\mathrm{PC}}$ as that found here there is no possibility of discriminating between the forms of the two $\varepsilon^{\prime}(\omega)$ curves using experimental data. Nevertheless, such agreement does not prove the correctness of Eqs. (2) and (4) or their appropriateness for CL situations when better alternatives are available.

The $\varepsilon_{\mathrm{CL}}^{\prime \prime}(\omega)=B_{0}$ real-part fit result is shown as the top dashed line in the figure. The estimated value of $\omega_{0}$ for the fit is about $2 \times 10^{139} \mathrm{r} / \mathrm{s}$, an exceptionally nonphysical value. In Sec. III A somewhat different results will be presented for full CNLS fitting of the present data using the Eq. 4 model, both without and with the addition of the effects of a parallel dielectric constant, $\varepsilon_{x}$, which can be taken as $\varepsilon_{\infty}$. Note that the present value of about 0.0435 differs somewhat from the value of 0.042 found in Ref. 3 and shown as the bottom dashed line in the figure. It is worth mentioning, however, that although the actual $\varepsilon^{\prime \prime}(\omega)$ data points included in Fig. 8 of Ref. 3 do not lie entirely on the $\varepsilon^{\prime \prime}(\omega)=B_{0}=0.042$ line, they certainly do not show consistent NCL power-law behavior and are characterized as being in excellent agreement with this constant value. ${ }^{3}$ Therefore, we shall consider that the $122 \mathrm{~K}$ data are well represented by the combination of the NCL $\varepsilon_{P C}^{\prime}(\omega)$ data and the $\varepsilon^{\prime \prime}(\omega)=0.042$ CL result, just as in Ref. 3. The resulting synthetic data set will be used below to evaluate the possibility of accurately fitting both its real and imaginary parts with composite models that are consistent with the KK relations. 


\section{B. Surprising series effects}

In order to represent possible electrode electrical effects we shall consider the simplest model, a CPE defined at the complex resistivity level. The result, termed the SCPE, may be written as

$$
\rho_{\mathrm{SC}}(\omega)=\left(\varepsilon_{V} A_{\mathrm{SC}}\right)^{-1}(i \omega)^{-\gamma_{\mathrm{SC}}}
$$

where $0<\gamma_{\mathrm{SC}} \leqslant 1$ and $A_{\mathrm{SC}}$ is an ideal dielectric constant, $\varepsilon_{S}$, when $\gamma_{\mathrm{SC}}=1$. Note that when used alone, this equation leads to a result fully equivalent to that of the PCPE of Eq. (3) when expressed at the complex conductivity or complex dielectric constant level. But the crucial point here is that the response defined by Eq. (5) is never alone but always in series with other response contributions; this makes a great difference as we shall see.

Although no CPE response can apply over the full frequency range, this is experimentally immaterial because other physically realizable processes will dominate the full response of the material at sufficiently high or low frequencies. We shall denote the combination of a $\mathrm{K} 1$ response model and the SCPE by K1S. Physically, series CPE response may arise from fractal effects in the electrodes, from nonideal diffusion, or from other causes. ${ }^{21}$ When nonparention electrodes are employed, the usual case in the present area, one would expect to observe blocking or nearly blocking behavior at sufficiently low frequencies. A SCPE is then a plausible choice since it leads to full blocking when $\gamma_{\mathrm{SC}}$ $=1$.

Consider the combination of a PCPE and a SCPE in series, where we identify the PCPE as associated with bulk response. In forming composite model designations, we shall always list bulk elements first and series ones to the right. Thus, the above composite model will be designated by PS. When the SCPE reduces to a pure frequency-independent capacitance, denote the result by PC; similarly when $\gamma_{\mathrm{PC}}$ $=0$, the result is termed the CS model. Note that electrically these composite models are fully equivalent, but they nevertheless differ in their physical interpretation. This distinction becomes particularly important in the usual case where the bulk response involves more than just the PCPE; for example, it might be include both the PCPE and the K1 in parallel, the PK1.

The CS model, with $\varepsilon_{x}=\varepsilon_{\infty}$, provides a simple illustration of some surprising effects. In practice, if the K1 model is found to best describe ionic dispersion at higher temperatures than those where NCL is dominant, we should expect its $\varepsilon_{C 1 \infty}$ contribution to $\varepsilon_{\infty}$ to be still present in the NCL region, and $\varepsilon_{x}$ should then be identified as $\varepsilon_{D \infty}$ for a CK1S model. A straightforward calculation, included below, shows that the CS series combination leads to terms in $\sigma^{\prime}(\omega)$ which exhibit power-law exponents (also termed $\log -\log$ slopes or just slopes) of $\left(2-\gamma_{\mathrm{SC}}\right), 1$, and $\gamma_{\mathrm{SC}}$. Because of the formal equivalence of the CS and PC models, for the PC the equivalent slopes become $\left(1+\gamma_{\mathrm{PC}}\right), 1$, and $\left(1-\gamma_{\mathrm{PC}}\right)$. Thus, behavior with $\left(2-\gamma_{\mathrm{SC}}\right)$ may appear in the lower frequency range and is then followed, without an appreciable frequency range with a slope of unity, by response involving an exponent value of $\gamma_{\mathrm{SC}}$. Now set $\delta=1-\gamma_{\mathrm{SC}}$ or $\gamma_{\mathrm{PC}}$; then one finds a slope of $(1+\delta)$ at low frequencies leading to (1

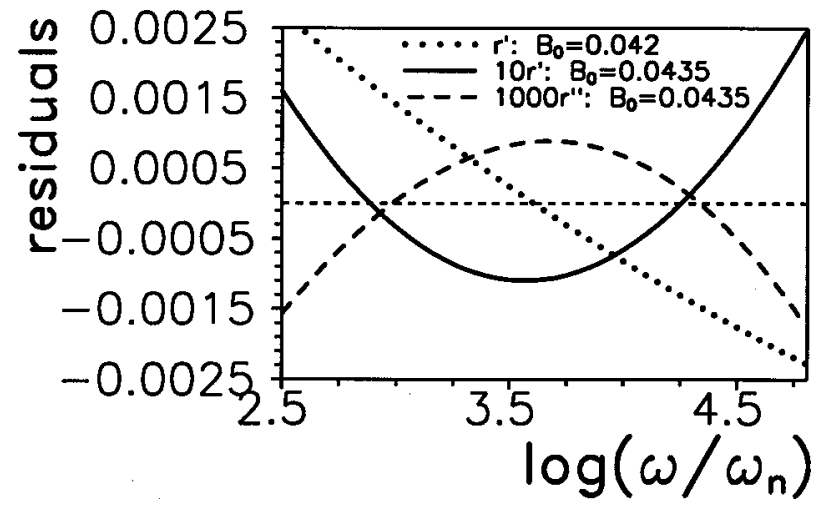

FIG. 2. Residuals $=$ (data values - fit estimates $)$ for the line 5 and line 6 CSC-K1 composite-model fits of Table I. Here $r^{\prime}$ and $r^{\prime \prime}$ designate real and imaginary part residuals, and the line 6 residuals are shown at 10 and 1000 times their actual values, respectively.

$-\delta$ ) in a higher frequency region. For small $\delta$, such response will not be experimentally distinguishable from a slope of unity, which, of course, leads to constant loss at the $\varepsilon^{\prime \prime}(\omega)$ level. Such apparent CL model response is, of course, consistent with the KK relations, unlike that associated with Eqs. (2) and (4).

It is straightforward to show that the CS model involving $\varepsilon_{\infty}$ leads to the following response at the complex dielectric constant level,

$\varepsilon_{C S}^{\prime \prime}(\omega)$

$$
=\frac{A_{\mathrm{SC}} \cos (\psi)}{\omega^{1-\gamma_{\mathrm{SC}}+\left(A_{\mathrm{SC}} / \varepsilon_{\infty}\right)^{2} \omega^{-\left(1-\gamma_{\mathrm{SC}}\right)}+2\left(A_{\mathrm{SC}} / \varepsilon_{\infty}\right) \sin (\psi)} .}
$$

If we now restrict consideration to NCL situations where the $\varepsilon$ " residuals involve a symmetrical convex curve such as that discussed later in Fig. 2, then we can pick frequencies $\omega_{1}, \omega_{m}$, and $\omega_{2}$ below the maximum, at the maximum, and above it, respectively. Select $\omega_{1}$ and $\omega_{2}$ so that $\varepsilon_{C S}^{\prime \prime}(\omega)$ is the same at these two frequencies. Then $\omega_{1} \omega_{2} \simeq \omega_{m}^{2}$ and one finds that the equality condition leads to $\left(A_{\mathrm{SC}} / \varepsilon_{\infty}\right) \simeq \omega_{m}^{\delta}$.

We may next rewrite Eq. (6) in the form

$$
\varepsilon_{C S}^{\prime \prime}(\omega) \simeq \frac{A_{S C} \sin (\delta \pi / 2)}{\omega^{\delta}+\omega_{m}^{2 \delta} \omega^{-\delta}+2 \omega_{m}^{\delta} \cos (\delta \pi / 2)} .
$$

Now for $\delta \ll 1$, on using the $A_{\mathrm{SC}} / \varepsilon_{\infty} \simeq \omega_{m}^{\delta}$ condition, we obtain

$$
\varepsilon_{C S}^{\prime \prime}(\omega) \simeq \frac{\varepsilon_{\infty}(\delta \pi / 2)}{\left[1+\left(\omega / \omega_{m}\right)^{\delta}\right]\left[1+\left(\omega / \omega_{m}\right)^{-\delta}\right]} .
$$

For small $\delta$, the $\omega$ terms in the denominator will be quite close to unity over the entire range, so on approximating them as unity, we finally obtain the approximate result

$$
\varepsilon_{C S}^{\prime \prime}(\omega) \simeq B_{0}=\varepsilon_{\infty}(\delta \pi / 8),
$$

which shows that the approximate $\mathrm{CL}, B_{0}$, depends directly on both $\varepsilon_{\infty}$ and $\delta$. This expression and that for $A_{\mathrm{SC}} / \varepsilon_{\infty}$ hold quite accurately when $\delta \leqslant 0.01$. Finally, note that because of the formal equivalence of the CS and PC models, Eq. (9) also applies for the latter model when $\varepsilon_{\infty}$ is replaced by $\varepsilon_{S}$, 
TABLE I. Table I. Results of CNLS fitting of synthetic NCL $\varepsilon(\omega)$ data. Here $S_{F R}$ and $S_{F I}$ are the relative standard deviations of the individual $\varepsilon^{\prime}$ and $\varepsilon^{\prime \prime}$ fit residuals, respectively. Line 1 shows the series SCPE and the parallel PCPE parameters for the exact $\varepsilon^{\prime}(\omega)$ input data. A constant value of $\varepsilon^{\prime \prime}(\omega)=0.042$ was used for all fitting results shown except the three that include an $\mathrm{X}$ after their row numbers. For these, a value of 0.0435 was employed. The B designation of row 5B indicates that the fitted data involved a wider frequency range than that used for the other fits. The number of free fitting parameters in a model is denoted by $n_{p}$. Composite model designations involve one or more bulk element names at the left, all in parallel electrically, and a final electrode-related series element designation, S, at the right. Here S indicates the SCPE; P the PCPE; an initial C, as in CS, specifies that the composite fitting model involves a frequency-independent bulk dielectric constant $\varepsilon_{x}$; a final $\mathrm{C}$, as in PK1C, indicates that the fitting model includes a dielectric constant $\varepsilon_{s}$, in series with all bulk elements; and K1 denotes the KWW1 model used in modulus-formalism fitting. Thus, CK1S denotes a K1 model in parallel with $\varepsilon_{x}$ and the result in series with a SCPE. All K1 parameters were fixed during the present fittings.

\begin{tabular}{|c|c|c|c|c|c|c|c|c|c|}
\hline No. & Model, $\mathrm{n}_{\mathrm{p}}$ & $10^{4} S_{F R}$ & $10^{4} S_{F I}$ & $10^{3}\left(1-\gamma_{\mathrm{SC}}\right)$ & $A_{\mathrm{SC}}$ or $\varepsilon_{s}$ & $10^{3} \gamma_{\mathrm{PC}}$ & $A_{\mathrm{PC}}$ or $\varepsilon_{x}$ & $\varepsilon_{C 1 \infty}$ & $\varepsilon_{\infty}$ \\
\hline 1 & $\mathrm{CPE}, 0$ & - & - & 3.20 & 8.885 & 3.20 & 8.885 & - & - \\
\hline 2 & CPE, 2 & 1.75 & 49.3 & 3.09 & 8.877 & 3.09 & 8.877 & - & - \\
\hline 3 & $\mathrm{PK} 1,2$ & 2259 & 1053 & - & - & 66 & 0.688 & 10.17 & 10.17 \\
\hline 4 & $\mathrm{~K} 1 \mathrm{~S}, 2$ & 35.1 & 226 & 20.4 & 67.24 & - & - & 10.17 & 10.17 \\
\hline 5 & $\mathrm{CS}, 3$ & 1.75 & 0.22 & 6.18 & 18.22 & 0 & 17.30 & - & 17.30 \\
\hline $5 \mathrm{X}$ & $\mathrm{CS}, 3$ & 0.12 & 0.23 & 6.40 & 18.25 & 0 & 17.30 & - & 17.30 \\
\hline $5 \mathrm{~B}$ & $\mathrm{CS}, 3$ & 4.61 & 0.96 & 6.13 & 18.07 & 0 & 17.45 & - & 17.45 \\
\hline 6 & CK1S, 3 & 1.76 & 0.17 & 6.19 & 18.26 & 0 & 7.10 & 10.17 & 17.27 \\
\hline $6 \mathrm{X}$ & CK1S, 3 & 0.12 & 0.19 & 6.42 & 18.29 & 0 & 7.10 & 10.17 & 17.27 \\
\hline 7 & PK1C, 3 & 1.75 & 1.58 & 0 & 11.83 & 16.8 & 25.3 & 10.17 & - \\
\hline $7 \mathrm{X}$ & PK1C, 3 & 0.12 & 1.70 & 0 & 11.83 & 17.4 & 25.5 & 10.17 & - \\
\hline
\end{tabular}

the electrode blocking dielectric constant, one that may usually be associated with a double- layer capacitance. Such a capacitance $^{21}$ is proportional to $T^{-1 / 2}$.

\section{COMPOSITE-MODEL FITTING RESULTS}

\section{A. Dominant NCL-CL situations}

Now consider the results of using composite models to fit the NCL and CL synthetic data derived from the $122 \mathrm{~K}$ $\mathrm{Na}_{2} \mathrm{O} \cdot 3 \mathrm{SiO}_{2}$ data in Ref. 3, discussed above. We shall begin by fitting using the Eq. (4) model with a dielectric constant, $\varepsilon_{x}$, in parallel with it. All of these CNLS fits yielded a virtually exact $B_{0}$ estimate of 0.0420 and $S_{F}$ values of about $10^{-4}$. Let $C_{0} \equiv \ln \left(\omega_{0} / \omega_{n}\right)$, where $\omega_{n}=1 \mathrm{r} / \mathrm{s}$. For $\varepsilon_{x}=0$, the results were similar to that already mentioned for real-part fitting and yielded $C_{0} \simeq 332$. No adequate fitting was possible when $B_{0}, C_{0}$, and $\varepsilon_{x}$ were all free. With $\varepsilon_{x}=7.1$ or 17.3, however, $C_{0} \simeq 66$ or -32 , respectively. Different results were found when a blocking dielectric constant, $\varepsilon_{S}$, was included in series with the Eq. (4) model. With a slightly larger value of $S_{F}$, the following estimates were found for $B_{0}, C_{0}$, and $\varepsilon_{S}: 0.0437,326$, and 443 , respectively, with the $\varepsilon_{S}$ value poorly determined. These results, particularly the $C_{0}$ estimates, indicate that the Eq. (4) model is inadequate.

Next, we shall consider physically realizable composite models possibly involving PCPE, SCPE, and K1 elements. The parameters of the K1 model were extrapolated to $122 \mathrm{~K}$ from those found at temperatures of $303 \mathrm{~K}$ and above using a relaxation-time activation energy of $0.7 \mathrm{eV}^{3,17}$ Although such a long extrapolation is sure to be very approximate, it turns out that its resulting $\varepsilon^{\prime \prime}(\omega)$ response at the lowest frequency present is more than one hundred times smaller than the $\varepsilon^{\prime \prime}(\omega)=0.042$ value, and since it decreases with a $\beta_{1}$ slope value of 0.34 , it is relatively even smaller at higher frequencies. Therefore, only the $\varepsilon_{C 1 \infty}$ associated with the model will have any appreciable effect. At $\mathrm{T}=303 \mathrm{~K}$, a good fit of the data using the CK1S model led to an $\varepsilon_{C 1 \infty}$ estimate of about 3.4. The value of this quantity calculated from the
$\mathrm{K} 1$ parameters extrapolated to $\mathrm{T}=122 \mathrm{~K}$ was 10.17 . Since there is good evidence that $\varepsilon_{C 1 \infty}$ is proportional to $1 / \mathrm{T}$ if $\beta_{1}$ is temperature independent, ${ }^{18}$ one should then expect a value of about 8.5 at $122 \mathrm{~K}$, somewhat smaller than 10.17. This difference is discussed below. In the fits involving K1, all three of its parameters were held constant.

The NCL parameter values defining the exact CPE response presented in Fig. 1 are listed in line 1 of Table I for comparison. We see that in line 2, as expected, simultaneous CNLS fitting of the NCL real-part data and the CL imaginary-part data leads to the same results for both SCPE and PCPE. Comparison with the line 1 results indicates that the parameter estimates are reasonable, but it is evident that the imaginary-part of the fit is far worse than the real-part one. This is because we are here fitting a power-law NCL model to CL response.

Rows 3 and 4 show the results of fitting with a PCPE in parallel with $\mathrm{K} 1$ and with $\mathrm{K} 1$ in series with an SCPE element. Since K1 response here is essentially only that of $\varepsilon_{C 1 \infty}=10.17$, these results are equivalent to those which would be obtained with $\mathrm{CP}$ and CS models with a fixed value of epsilon of 10.17. Note that the fitting accuracy of rows 3 and 4 is very poor, but that for row 3 is much worse than that of row 4. In fact, fits of the CP model with $\varepsilon_{x}$ free to vary led to its approach to zero, the line 2 result.

The results of line 5 fitting with the CS model, a SCPE in series with a dielectric constant, show that the imaginary part fit is now more than 200 times better than those involving only a CPE, although the SCPE parameter estimates are appreciably different from those in line 1 . It is therefore clear that the two slopes present with this combination, as discussed in the last section, can indeed allow exceptionally close fitting of actual CL response.

The proper interpretation of experimental data similar to that considered here is that, while true CL response is nonphysical, a model which involves a CPE in series with other response elements can lead to an apparent CL response which cannot be distinguished from true $\mathrm{CL}$ for such data. As 
already mentioned, since the CS and PC models are electrically equivalent, it is unnecessary to show PC results in Table I. But this equivalence means that fitting results of NCL-CL response with a CPE and capacitance in series may be interpreted as involving NCL bulk response in series with a blocking electrode, as in the PC model, or as that of a bulk capacitance, say $\varepsilon_{\infty}$, in series with NCL electrode effects. It is probable that the first choice is the appropriate one for most situations.

As a test of the stability and consistency of row 5 fitting results, line $5 \mathrm{~B}$ presents the results of a $\mathrm{CS}$ fit using data exactly like those of lines 1 and 5 except extending over the much larger range from 1 to $10^{5} \mathrm{r} / \mathrm{s}$. Although the relative standard deviations of the residuals are larger, the fit is still excellent and the parameter estimates are comparable. Further, comparison of the line 5 and line $5 \mathrm{X}$ results show that the real part fit is much superior for the latter, the situation where $B_{0}$ is the average of the power-law $\varepsilon^{\prime \prime}(\omega)$ CPE data of row 1.

Rows 6 and $6 \mathrm{X}$ show results when the composite fitting model involves a SCPE in series with the parallel combination of the bulk $\varepsilon_{x}$ and a conductive-system K1 bulkresponse. As expected, they are nearly identical to those in rows 5 and $5 \mathrm{X}$. But note that the CK1 combination corresponds to the CMF situation, one that has been shown to fit much data better and more consistently than the K1 OMF approach, ${ }^{9,16,17}$ here represented by the K1S results of row 4 . The CK1S model is included here because, unlike the K1S, $\mathrm{CS}$, or PC models it leads to a separate estimate of $\varepsilon_{x}$ $=\varepsilon_{D \infty}$. Such models as CS or PC yield only an estimate of $\varepsilon_{x}$ equal to $\varepsilon_{\infty}=\varepsilon_{C 1 \infty}+\varepsilon_{D 1 \infty}$.

Notice that comparison of the line 4 and line 6 results indicates that the K1S composite model yields a much poorer fit than does the CK1S. This is clearly because an $\varepsilon_{\infty}$ value of about 17.30 is needed, appreciably larger than the $\varepsilon_{C 1 \infty}$ value provided by the $\mathrm{K} 1 \mathrm{~S}$ model. This conclusion is verified by first changing the characteristic relaxation time of the $\mathrm{K} 1$ model by a small factor so that $\varepsilon_{C 1 \infty} \simeq 17.3$ and then repeating the K1S fit. One finds almost exactly the same results as those of line 5 except that $10^{4} S_{F I}$ changes to 0.32 , still an excellent fit.

The results of lines 6 and $6 \mathrm{X}$ show that the expected value of $\varepsilon_{\infty}$ is found when the free fitting parameter $\varepsilon_{x}$ is added to the composite model. This quantity should then estimate $\varepsilon_{D \infty}$, and we see that its value of 7.10 is only slightly larger than the $303 \mathrm{~K}$ estimate of about 6.8. But note that if instead of the constant value $\beta_{1}=0.34$ used in the $\mathrm{K} 1$ extrapolation one uses a value of 0.36 , it turns out that $\varepsilon_{C 1 \infty}$ is then about 8.34 and the $\varepsilon_{x}=\varepsilon_{D_{\infty}}$ estimate becomes 8.95 , with the other parameters remaining nearly the same as those of line 5. The $\varepsilon_{C 1 \infty}$ value is much closer to its expected value of 8.5 and the increased value of $\varepsilon_{D \infty}$ is still not unreasonable. These results show that the same fitting model may be consistently used for both $303 \mathrm{~K}$ and $122 \mathrm{~K}$ and that the present low-temperature estimates of $\varepsilon_{C 1 \infty}$ and $\varepsilon_{D \infty}$ are plausible and self-consistent.

Comparison of the line 5 and line 6 results shows that the only significant change on fitting with $\varepsilon^{\prime \prime}(\omega)=0.042$ or 0.0435 is a large reduction in $10^{4} S_{F R}$ for the latter choice, not surprising since Fig. 1 indicates that 0.0435 is the best constant approximation to the $\varepsilon_{P C}^{\prime \prime}(\omega)$ NCL response shown in the figure. Figure 2 shows some line 5 and line 6 fitting residuals. It is clear that the change from $B_{0}=0.0420$ to $B_{0}$ $=0.0435$ not only reduces the $\varepsilon^{\prime}(\omega)$ residuals greatly, but changes their general character as well. The very small $\varepsilon^{\prime \prime}(\omega)$ residuals, essentially the same for both $B_{0}$ choices, show both how close the fit is to CL behavior and, as well, demonstrate that while it could not be distinguished from exact CL response, it is nevertheless not CL.

Lines 7 and $7 \mathrm{X}$ in the table present PK1C fitting results, ones that show appreciable differences from the corresponding row 6 and $6 \mathrm{X}$ CK1S results. In particular, we see that the imaginary-part fit quality factor, $10^{4} S_{F I}$, is about ten times larger for the PK1C results. Further, the NCL exponent $\gamma_{\mathrm{PC}}$ is nearly three times larger than the $\left(1-\gamma_{\mathrm{SC}}\right)$ factor, so PK1C yields a poorer approximation to true CL than does the CK1S model. It follows that $A_{\mathrm{PC}}$ is not very close to a pure dielectric constant quantity and thus cannot be identified as a reasonable approximation to $\varepsilon_{D_{\infty}}$, precluding estimation of $\varepsilon_{\infty}$.

Although the CK1S is clearly superior to the PK1C for the present semi-synthetic data, different results may be found in fitting some actual low-temperature data showing NCL-CL behavior. Therefore, it would be appropriate to investigate the utility of both models in such situations. Finally, it is worth mentioning that for the present data, fits with the PS and PK1S models, which involve four free parameters, result in so large uncertainties in the values of one or two of the parameters that the fits must be rejected. The PK1S model is a generalization of the CK1S one since it reduces to the latter when $\gamma_{\mathrm{PC}} \rightarrow 0$. Thus, for sufficiently small $\gamma_{\mathrm{PC}}$ values, both models are of CMF character, with $A_{\mathrm{PC}}$ thus approximating the $\varepsilon_{x}=\varepsilon_{D_{\infty}}$ of the CK1S model.

\section{B. Higher-temperature NCL contributions}

The $-0.5^{\circ} \mathrm{C}, \mathrm{Na}_{2} \mathrm{O} \cdot 3 \mathrm{SiO}_{2}$ data previously analyzed in terms of a CL by $\mathrm{Ngai}^{1}$ will be fitted here by a different approach in order to further investigate the presence of NCL or CL behavior in the data. Now define the electric modulus $M(\omega) \equiv i \omega \varepsilon_{V} \rho(\omega)=1 / \varepsilon(\omega)$. Figure 3 shows the $M^{\prime \prime}(v)$ data for this set and also the results of a K1 OMF fit to it. Here unity weighting ${ }^{20}$ (UWT) was used to emphasize the largest values in the data at the expense of the smaller ones. Note that when the K1S model was used, again with unity weighting, an estimate of $\beta_{1}=0.47$ was found, in agreement with that found by Ngai, possibly by using a table of the width of the curve at half height, ${ }^{15}$ and also equal to that cited earlier in Ref. 23 by Moynihan. Ngai fitted $M^{\prime \prime}$ data without including the effect of the original high-frequency $M^{\prime \prime}$ points and calculated the corresponding $\sigma^{\prime}(v)$ curve. He then showed that the difference between this result and the original $\sigma^{\prime}(v)$ data led to points which fell closely on a line involving $v^{1.0}$ behavior, an indication of pure CL behavior.

Here the data have been fitted by both the PK1S and CK1S models with the usual proportional weight (PWT) choice and seven and six free parameters, respectively. Although it has been found that fits of the same data for differ- 


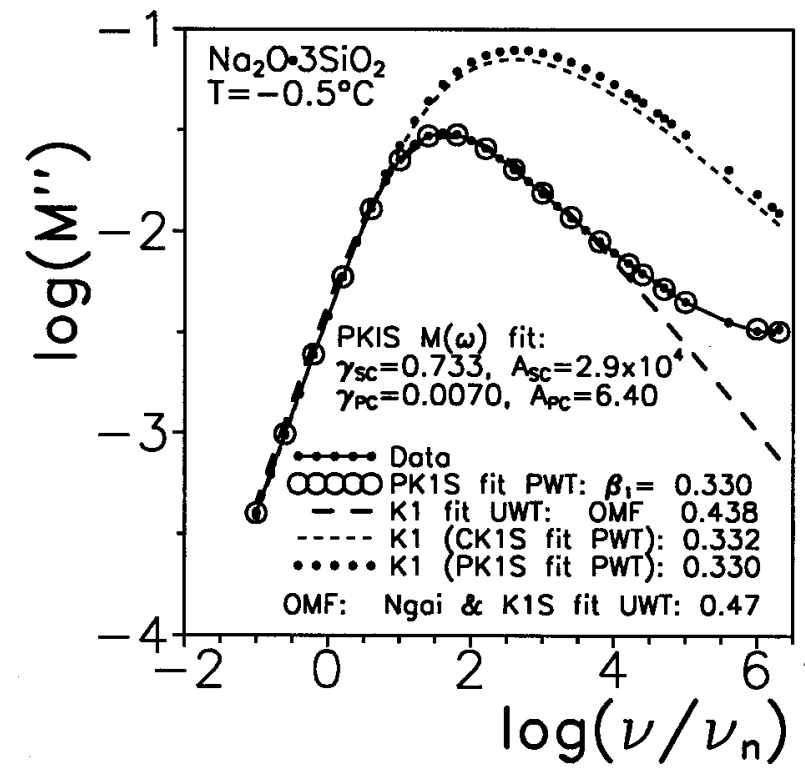

FIG. 3. $\log -\log$ plots of $M^{\prime \prime}(v)$ data and fits vs frequency $v$. For clarity, only every other PK1S fit point is included. Here and elsewhere $v_{n}=1 \mathrm{~Hz}$.

ent materials with these two models often yield closely comparable fits, here $S_{F}$ was about 0.018 for the PK1S and about 0.031 for the CK1S, an immediate indication of the presence of NCL behavior associated with the PCPE. Further, the PK1S fit results in the figure show that it is important to include electrode effects modeled by the SCPE. Here its estimated parameter values were closely the same for both fits. Note that since $\left(2-\gamma_{\mathrm{SC}}\right)$ was about 1.3 , it is evident that here the SCPE must represent electrode effects rather than NCL behavior. In fact, the slope of the full $\sigma^{\prime}(v)$ data was found to increase continuously with frequency up to a maximum slightly larger than unity with no evidence of either an approach to saturation or a decrease, thus almost certainly electrode dominated at the high frequency end.

Figure 3 also shows K1-only curves calculated from the PK1S and CK1S fits. We see that they are close to each other and both involve $\beta_{1}$ values of about 0.33 , far different from the OMF value of about 0.47 . Since a value of about 0.33 is also found for direct fitting of the full $\sigma^{\prime}(v)$ data, where $\varepsilon_{x}=\varepsilon_{D_{\infty}}$ plays no significant role, it is evident that the absence of such a term in OMF fitting approaches yields ${ }^{9,16-18,23}$ incorrect estimates of $\beta_{1}$.

Figure 4 presents $\varepsilon(v)$ results for the present data set, results somewhat comparable to those of Ngai. ${ }^{1}$ Here, however, we distinguish between Ngai's CL, which made use of the inappropriate Eq. (2), and the present NCL behavior involving a $\sigma^{\prime}(v)$ slope of $(0.993 \pm 0.0005)$ instead of 1.0. The contributions of the bulk PCPE term of the PK1S model are shown separately on the figure as well as the K1S response without NCL effects. Estimates of $\varepsilon_{D \infty}$ and $\varepsilon_{\infty}$ are about 5.9 and 8.4, respectively. Both values are physically and statistically significant and cannot be separately estimated by OMFtype analysis. The rapid rise in $\varepsilon^{\prime}(v)$ at the lowest frequencies is associated with SCPE electrode effects. It is thus evident that for the present data, and for most similar data, electrode effects play a significant role both at both the low

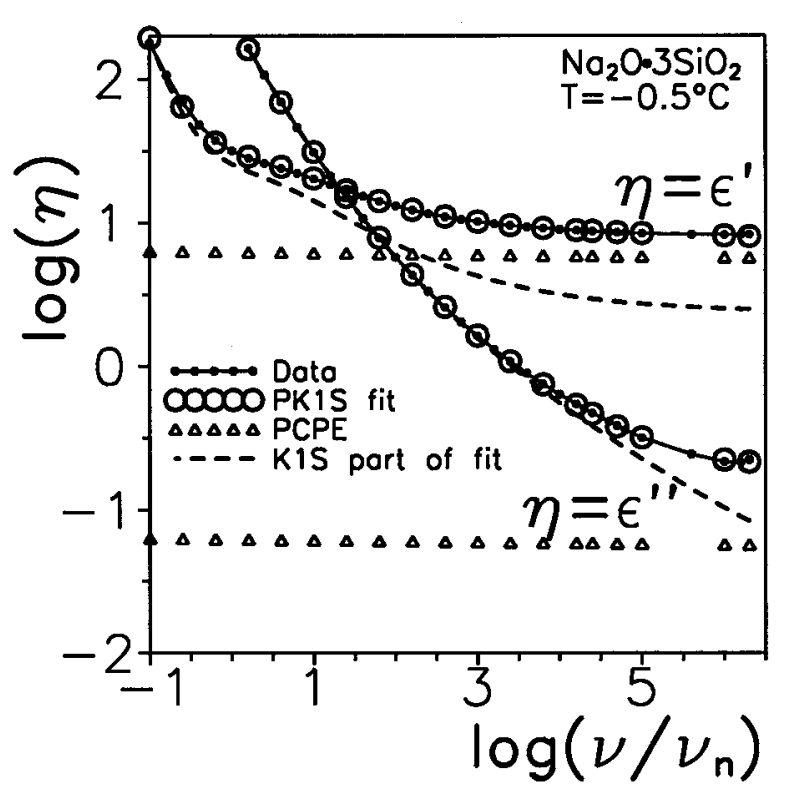

FIG. 4. $\log -\log$ plots of $\varepsilon^{\prime}(v)$ and $\varepsilon^{\prime \prime}(v)$ data and fits vs frequency.

and high frequency ends of the range and clearly should not be neglected in data fitting and analysis.

\section{DISCUSSION}

For the first time, it has been possible to find physically realistic models that lead to excellent fits and to significant parameter estimates for complex data of the present NCL-CL type. In the past, NCL fitting models have mostly been restricted to such forms as the $A \omega^{S}$ term in Eq. (1) and have thus not been concerned with its imaginary part KK pair. ${ }^{1,3,7,8}$ Even those approaches which use the present Eq. (2) to take some account of both real and imaginary parts of data ${ }^{1,3}$ do not employ CNLS fitting of both parts simultaneously. The use of a CPE in series with bulk response has been shown here to provide both excellent CNLS fits and significant parameter estimates.

The estimated $\varepsilon_{\infty}$ values, as presented in lines $5,5 \mathrm{X}, 5 \mathrm{~B}$, 6 , and $6 \mathrm{X}$ of Table I, are particularly noteworthy because they are consistent with values found at higher temperatures where NCL effects are dominated by other responses when the higher-temperature values are extrapolated to $122 \mathrm{~K}$. No such consistent estimates have been presented earlier. Equations (6)-(8), and especially the well-fitting Eq. (9), show that the apparent CL quantity $B_{0}$ may be directly related, for CS or PC model fitting, to the product of $\varepsilon_{x}$ and $\delta$, or $\varepsilon_{S}$ and $\delta$.

Although Ngai has pointed out that NCL behavior may appear in pure structures containing no ions and few impurities, ${ }^{1,24}$ providing a possible reason for the identification of the present NCL-CL behavior with bulk dielectric dispersion, such a conclusion seems inconsistent with the present CS model, but not necessarily with the PC one where NCL-CL is a bulk rather than an electrode phenomenon. In contrast, although the CS model leads to excellent CNLS data fits and plausible parameter estimates, it seems to require that the SCPE be associated with processes at the material-electrode interface and even within the electrode. 
$\mathrm{Ngai}^{1}$ has discussed in detail the dependence of $B_{0}$ (often called $\Delta \varepsilon^{\prime \prime}$ ) on various effects related to localized ions in the material but has concluded ${ }^{24}$ that the physical origin of the constant loss is still not clear. If it were indeed associated with motion of ions across the interface, one might expect it to exhibit an activation energy comparable to that of the dc conductivity, but generally $B_{0}$ exhibits a much weaker dependence and seems to increase with increasing temperature with power-law or non-Arrhenius exponential behavior. ${ }^{1,6}$

The present NCL-CL fitting results, involving data for a single temperature, do not provide information on the temperature dependence of $B_{0}$, but they nevertheless suggest through Eq. (9) that it should be correlated with the product of a power-law exponent and $\varepsilon_{\infty}$, another quantity which shows only weak dependence on temperature, or possibly even with the double-layer blocking quantity $\varepsilon_{S}$ which is proportional to $T^{-1 / 2}$. Since the CS and PC models appear not to lead to $B_{0}$ temperature dependence closely comparable to experimental results, it seems likely that most lowtemperature data are of NCL-NCL rather than NCL-CL character. Then, for example, the PK1S model will be more appropriate. Accurate fitting of such data is needed to resolve the matter.

For the K1 CMF response model, at sufficiently high frequencies conductive-system ionic response leads to $\varepsilon_{C 1}^{\prime}(\omega) \rightarrow \varepsilon_{C 1 \infty}$, where $\varepsilon_{C 1 \infty}$ is most likely associated with short-range librational and vibrational motion of ions. But since the associated $\varepsilon_{C 1}^{\prime \prime}(\omega)$ quantity in the range where $\varepsilon_{C 1 \infty}$ is essentially frequency independent does not exhibit NCL power-law response, as discussed above, it seems unlikely that such intensive bulk behavior should be identified with NCL response of either of the above types. If so, it seems more plausible that any data well fitted by the CS model indeed involves ionic motion across the materialelectrode interface, perhaps potentiated by stress in this region.

Finally, both the CS and PC models can lead to real-partconductivity slopes appreciably greater than unity, often ap- parent in the high frequency region. Discussion of such observations, especially at very high frequencies, appears in the present Ref. 25 and a PK1S fitting example is discussed in the last section.

${ }^{1}$ K. L. Ngai, J. Chem. Phys. 110, 10576 (1999).

${ }^{2}$ A. S. Nowick, A. V. Vaysleyb, H. Jain, and X. Lu, Mater. Res. Soc. Symp. Proc. 411, 99 (1996).

${ }^{3}$ A. S. Nowick, A. V. Vaysleyb, and W. Liu, Solid State Ionics 105, 121 (1998).

${ }^{4}$ A. K. Rizos, J. Alifragis, K. L. Ngai, and P. Heitjans, J. Chem. Phys. 114, 931 (2001).

${ }^{5}$ A. Rivera, J. Santamaria, and C. León, Appl. Phys. Lett. 78, 610 (2001).

${ }^{6}$ C. León, A. Rivera, A. Várez, J. Sanz, J. Santamaria, and K. L. Ngai, Phys. Rev. Lett. 86, 1279 (2001).

${ }^{7}$ D. L. Sidebottom, Phys. Rev. B 61, 14507 (2000).

${ }^{8}$ W. K. Lee, J. F. Liu, and A. S. Nowick, Phys. Rev. Lett. 67, 1559 (1991).

${ }^{9}$ J. R. Macdonald, Solid State Ionics 133, 79 (2000).

${ }^{10}$ J. R. Macdonald, J. Appl. Phys. 75, 1059 (1994).

${ }^{11}$ J. R. Macdonald, J. Non-Cryst. Solids 210, 70 (1997).

${ }^{12}$ A. Hunt, J. Phys.: Condens. Matter 4, 5371 (1992); J. Non-Cryst. Solids 160, 183 (1993).

${ }^{13}$ A. S. Nowick, A. V. Vaysleyb, and I. Kuskovsky, Phys. Rev. B 58, 8398 (1998).

${ }^{14}$ C. J. F. Böttcher and P. Bordewijk, Theory of Electric Polarization, Vol. II (Elsevier, Amsterdam, 1978), p. 58.

${ }^{15}$ C. T. Moynihan, L. P. Boesch, and N. L. Laberge, Phys. Chem. Glasses 14, 122 (1973).

${ }^{16}$ J. R. Macdonald, J. Non-Cryst. Solids 197, 83 (1996).

${ }^{17}$ J. R. Macdonald, J. Non-Cryst. Solids 212, 95 (1997).

${ }^{18}$ J. R. Macdonald, Phys. Rev. B 63, 052205 (2001).

${ }^{19}$ R. Kohlrausch, Ann. Phys. (Leipzig) 91, 179 (1854); G. Williams and D. C. Watts, Trans. Faraday Soc. 66, 80 (1970).

${ }^{20}$ J. R. Macdonald and L. D. Potter, Jr., Solid State Ionics 23, 61 (1987); J. R. Macdonald, J. Comput. Phys. 157, 280 (2000). The newest version of the comprehensive LEVM fitting program may be downloaded at no cost from http://www.physics.unc.edu/ macd/. It includes an extensive manual, executable programs, and full source code. More information is provided about LEVM at this www address.

${ }^{21}$ Impedance Spectroscopy - Emphasizing Solid Materials and Systems, edited by J. R. Macdonald (Wiley-Interscience, New York, 1987).

${ }^{22}$ J. R. Macdonald, Solid State Ionics 13, 147 (1984).

${ }^{23}$ C. T. Moynihan, Solid State Ionics 105, 175 (1998).

${ }^{24}$ K. L. Ngai, J. Non-Cryst. Solids 274, 155 (2000).

${ }^{25}$ J. R. Macdonald, J. Appl. Phys. 90, 153 (2001). 